\title{
Pulse compression and traveling wave excitation scheme using a single dispersive element
}

\author{
Sandor Szatmari, Gotz Kuhnle, and Peter Simon
}

\begin{abstract}
A single dispersive element is shown to be sufficient for simultaneous pulse compression and tilting of the pulse front, and therefore well-suited for traveling-wave excitation of targets. It is shown that in all the previous arrangements used for traveling wave excitation, spatially dependent group velocity dispersion occurs along the target. A compensated arrangement is proposed that provides pulse compression at the target-plane and exact synchronism between the pump and the generated pulses for various targets.
\end{abstract}

\section{Introduction}

Traveling wave excitation (TWE) is of great importance in the field of short pulse generation ${ }^{1-19}$ and as shown recently in laser-induced plasma and $\mathrm{x}$-ray laser experiments. ${ }^{20-23}$ Longitudinal pumping is a commonly used technique for TWE of amplified spontaneous emission (ASE). ${ }^{1-10}$ With picosecond excitation, the duration of the TWE-ASE pulse is 3-10 times the duration of the pump pulse achieved in these experiments.

In Refs. 11 and 12, a transversal pump scheme is introduced for TWE-ASE, providing pulses with improved parameters. The key element of this arrangement is a diffraction grating, used before the dye cell to create a continuous temporal delay across the diffracted pump beam (Fig. 1). If $\tan \gamma=\eta$ is fulfilled, where $\gamma$ is the tilt angle of the pulse front as shown in the figure, $\eta$ is the group velocity index of the dye solution at the wavelength of ASE radiation to be generated, the pump and the ASE pulses are always in synchronism at any part of the excited volume, i.e., TWE-ASE is expected. Reference 11 gives a detailed comparison of the longitudinal and transversal pump schemes. It is found that besides the technical advantages of transversal pumping (less sensitivity for spatial inhomogeneities of pumping, less probability of self-focusing) there are physical limitations (related to the achievable gain and to synchronism between the generated and the pump pulse) which cannot be overcome by

Peter Simon is with Laser-Laboratorium Gottingen e. V., $21 \mathrm{Im}$ Hassel, D-3400 Gottingen, Federal Republic of Germany; the other authors are with Max Planck Institute for Biophysical Chemistry, Laser Physics Department, Postfach 28 41, D-3400 Gottingen, Federal Republic of Germany.

Received 26 September 1989.

0003-6935/90/365372-08\$02.00/0.

(C) 1990 Optical Society of America. longitudinal pump schemes, but only using transversal pumping. The experimentally observed shortening of the TWE-ASE pulse with respect to the pump pulse in Ref. 11 is explained as the result of the above-mentioned advantages of transversal pumping. These observations were later confirmed by computer calculations ${ }^{15,18}$ and were extended to different experimental realizations, ${ }^{13,14,16,17}$ and to shorter time scales. ${ }^{19}$

In Ref. 11, the transversal TWE pump scheme is regarded to provide exact synchronism between the ASE and the pump pulse, without considering additional unwanted effects leading to eventual temporal broadening of these pulses. However, this aproach is only valid for the picosecond time scale, ${ }^{11-18}$ or for very short pumped volumes. ${ }^{19}$

The problems are more stringent when the pump pulses are shorter in duration and of shorter wavelength and when the structure to be pumped must be relatively long. These are just the experimental conditions for how many $x$-ray laser experiments should be performed, and where the transversal TWE scheme is the only solution to expect lasing from short-living states. $^{20-23}$ Recent development of high power, $\mathrm{KrF}$ laser systems made it possible to generate $\sim 500-\mathrm{fs}$ chirped pulses at $248 \mathrm{~nm}$, which are compressible down to $\sim 100 \mathrm{fs},{ }^{24-26}$ using the refractive variant ${ }^{27,14}$ of the well-known Treacy compressor. ${ }^{28}$ It is shown in recent publications ${ }^{29,30}$ that spatially dependent distortion of the pulse front and the pulse duration occurs when a large aperture, femtosecond, short wavelength laser beam is sent through any optical component having chromatic aberration. Since many of the target experiments are planned to be performed with shortpulse excimer-laser excitation, it is necessary to study the limitations of TWE when a femtosecond, largeaperture laser beam of short wavelength is used as a pump pulse.

The earlier practice was that TWE schemes and pulse compressors were used separately ${ }^{22}$; their common feature has not been recognized. On the other hand, no care has been taken of the spatially depen- 


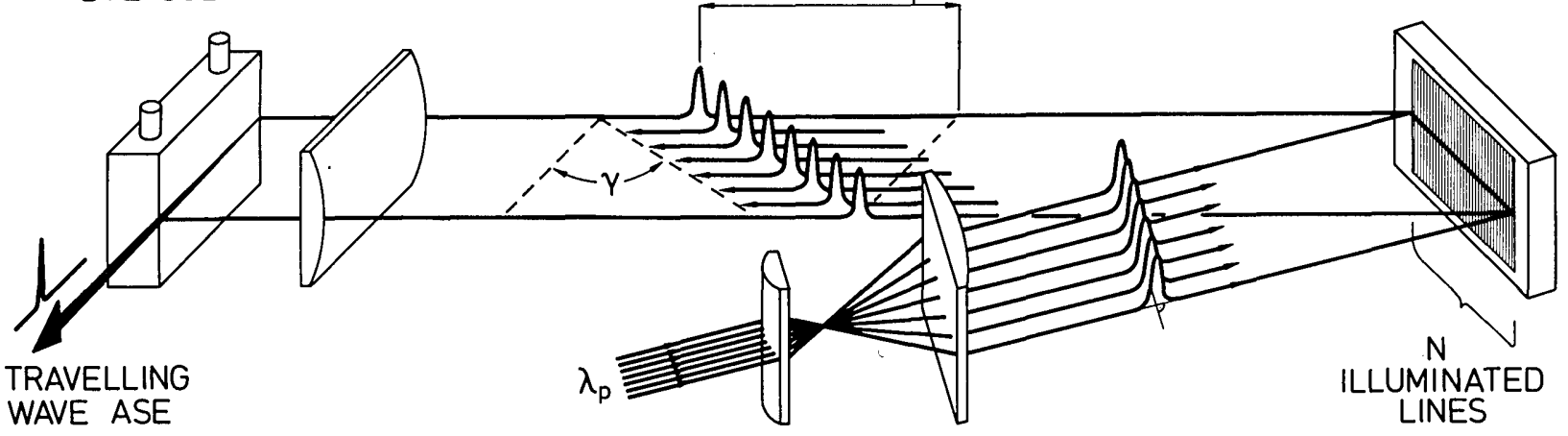

Fig. 1. Arrangement used earlier for transversal traveling wave excitation (taken from Ref. 11).

dent negative group velocity dispersion (GVD) of transversal TWE schemes, ${ }^{11-19}$ which we found had an inherent property that leads to spatially dependent pulse broadening at the target.

Regarding the above considerations, in this paper we concentrate on the following aspects of femtosecond TWE:

(1) exact synchronism of the pump pulse to the generated pulse;

(2) pulse compression of the pump pulse to get minimum pulse duration at the target; and

(3) avoidance of spatially dependent distortion of the pulse front and pulse duration.

\section{Limitations for the Different TWE Schemes}

It can be shown by simple considerations that longitudinal pumping has strong limitations for length $L$ of the pumped volume, if pulses of subpicosecond duration $\tau$ are used for TWE. The main requirement to be considered is synchronism between the pump and the generated pulse, which is necessary to get short TWEASE pulses. ${ }^{15}$ In case of no synchronism the time difference $\Delta \tau$ between the pump and the generated pulse is given by the equation

$$
\Delta \tau=\frac{L}{c}\left(\eta_{L}-\eta_{p}\right) \text {, }
$$

where $c$ is the speed of light, $\eta_{p}$ and $\eta_{L}$ are the group velocity indices of the pumped volume at the pump and the lasing wavelengths, respectively. If we require $\Delta \tau$ to be comparable with or smaller than $\tau(\Delta \tau<\tau)$, for a $\lambda_{p}=248 \mathrm{~nm}, \tau=100-\mathrm{fs}$ pump pulse and for a $\lambda_{L}=$ $340-\mathrm{nm}$ radiation to be generated in ethanolic solution, synchronism is fulfilled for $L<0.3 \mathrm{~mm}$ only. There are other limitations for $L$, related to temporal broadening of the pump pulse and of the pulse to be generated, resulting from GVD of the material, but all these are less important.

At first glance, these problems are solved when using a grating for transversal TWE-ASE, as suggested in Ref. 11. However, other limitations occur as are discussed in the following. Figure 2(a) shows a grating put into a nonchirped beam, i.e., all the spectral com- ponents are in temporal overlap before the grating, as shown schematically for two wavelength components in the figure (the red component is shown by a dashed line, the blue component by a solid line). After diffraction, because of the angular dispersion of the grating given by

$$
\frac{d \epsilon}{d \lambda}=\frac{1}{d \cos \beta},
$$

where $d$ is the grating constant and $\beta$ is the diffraction angle, the different spectral components are separation in a direction perpendicular to the propagation by

$$
\Delta=l \frac{d \epsilon}{d \lambda} \Delta \lambda,
$$

where $\Delta \lambda$ is the wavelength difference between the two spectral components, and $l$ is the distance measured from the grating, as shown in Fig. 2(a). Since the pulse front is tilted with respect to the phase front, where the tile angle $\gamma$ can be calculated from the equation

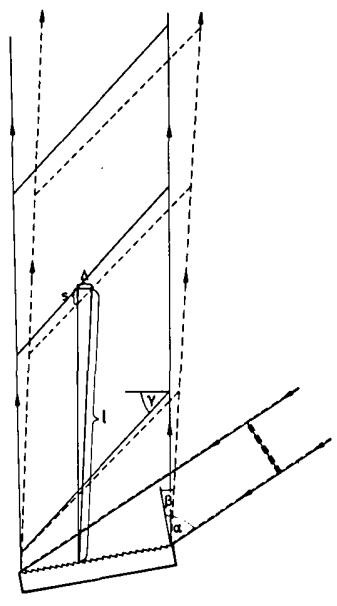

a)

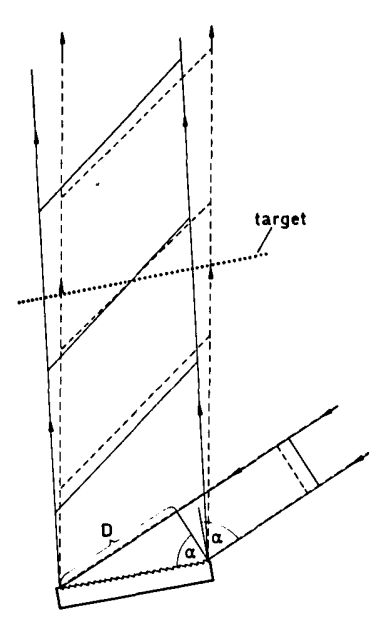

b)
Fig. 2. Schematic of the pulse fronts of the red (dashed line) and the blue component (solid line) of (a) a nonchirped and (b) a positively chirped pulse after diffraction on a grating. 


$$
\tan \gamma=\lambda \frac{d \epsilon}{d \lambda}
$$

as shown in Ref. 14, the above displacement $\Delta$ of the spectral components leads to spatial separation of the pulse fronts of the different spectral components. Since the displacement of spectral components $\Delta$ and separation $s$ of the pulse fronts in the direction of propagation, connected by

$$
s=\Delta \tan \gamma
$$

are linear functions of $l$, the negative chirp introduced by the grating is spatially dependent, and also a linear function of $l$, as seen by substituting Eqs. (3) and (4) into Eq. (5):

$$
s=l \lambda\left(\frac{d \epsilon}{d \lambda}\right)^{2} \Delta \lambda \text {. }
$$

From Eq. (6) one can obtain the temporal dispersion constant as

$$
\frac{d T}{d \lambda}=\frac{s}{c \Delta \lambda}=\frac{l \lambda}{c}\left(\frac{d \epsilon}{d \lambda}\right)^{2}
$$

This means that, if a pulse duration measurement of an originally unchirped pulse is carried out after a grating, a negatively chirped pulse is seen-and depending on separation $l-a$ longer pulse duration must be measured.

\section{Single Component Compressor and TWE Arrangement}

Comparing Eq. (7) to the temporal dispersion constant of the Treacy compressor ${ }^{28}$ or the double prism pulse compressor, ${ }^{14}$ one finds exact agreement with the result obtained in Ref. 14, where both dispersive components were considered for the calculations. This agreement means that the group velocity dispersion is already introduced by the first dispersive element of the compressor, and the second-identical, but oppositely positioned-dispersive element is only necessary to get again a pulse front perpendicular to the direction of propagation and to preserve the actual value of the chirp which belongs to that point in space, where the second dispersive element is located. This means that by the use of a single dispersive element in a properly chosen position, one can tilt the pulse front and can simultaneously introduce a negative GVD.

A practical example is shown in Fig. 2(b), where a single grating arrangement is presented for pumping a target with positively chirped input pulses, wherebecause of the negative GVD of this device-pulse compression is expected. The target [dotted line in Fig. 2(b)] is put in a position where the pulse fronts corresponding to different wavelengths are overlapping, so that the target sees a compressed pulse duration and at the same time a tilted pulse front. These considerations are only valid for the middle part of the beam, where all the spectral components are present (see Fig. 2). The margins having a width of $\sim \Delta$ can be blocked by a beam s:op. (This manipulation does not change the picture as long as $\Delta \ll L$, where $L$ is the length of the target.) This means that a second dis-

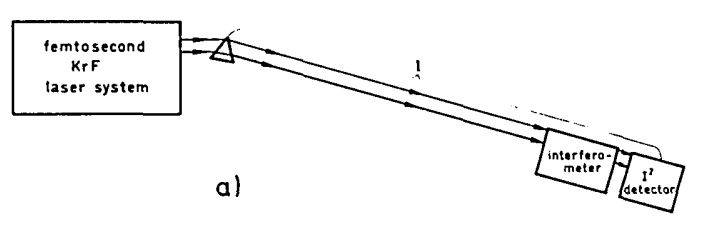

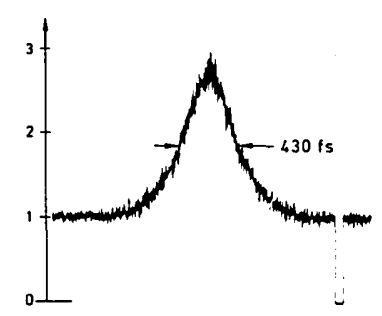

b)

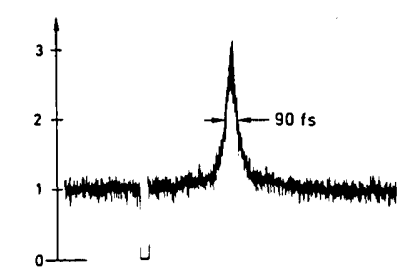

c)
Fig. 3. (a) Experimental arrangement used to study the operation of the single prism compressor. Autocorrelation traces obtained with (b) $1=0$ and (c) $1=280-\mathrm{cm}$ separation between the prism and the autocorrelator.

persion element is not necessary if temporal compression of pulses used for TWE is needed, in spite of the earlier practice (see, for example, Ref. 19). The scheme shown in Fig. 2(b) can also be used for experiments where a pulse front tilt is not necessary but can be tolerated, e.g., in experiments where there is no need to focus the beam, but where at each point of the target the shortest possible (compressed) pulse duration is required (power dependent transmission measurements, etc.). Even pump and probe experiments can be performed using this single component pulse compressor if both the pulse and the probe beams have the same tilt angle of the pulse fronts. In this way, the efficiency of pulse compression can be improved significantly.

\section{Experimental}

The above considerations were checked in an experiment where output pulses of a femtosecond $\mathrm{KrF}$ laser system were sent through a single dispersive element, and the pulse duration was monitored by an autocorrelator. The experimental arrangement is shown in Fig. 3(a). The femtosecond $\mathrm{KrF}$ laser system (built at Laser-Laboratorium Gottingen) is an improved version of that described in Ref. 25, delivering $\sim 10-\mathrm{mJ}$, $\sim 500$-fs chirped pulses at $248 \mathrm{~nm}$ with a repetition rate up to $20 \mathrm{~Hz}^{31}$ The output is sent through a $60^{\circ} \mathrm{LiF}$ prism. The autocorrelator is positioned after the prisms and the distance $l$ between the prism and the autocorrelator is varied. Before the autocorrelator a 4-mm diam pinhole is used to select the middle part of the beam that has $8-\times 22-\mathrm{mm}^{2}$ cross section with the longer axis lying horizontally. It is already discussed in Ref. 29 that autocorrelators, incorporating standard Michelson interferometers, are insensitive to the shape and the angle of the pulse front. They actually measure the temporal width of the pulse front, whatever its form is. One aspect to be considered when measuring the duration of a pulse having a strongly 
tilted pulse front is that the direction of displacement of the two partial beams is parallel to the direction of propagation, therefore enclosing an angle of $90^{\circ}-\gamma$ to the pulse fronts. Then measured pulse width $\tau^{\prime}$ is somewhat longer than pulse width $\tau$ that can be obtained if the pulse front is perpendicular to the direction of propagation as given by

$$
\tau^{\prime}=\frac{\tau}{\cos \gamma} .
$$

The autocorrelator used in these experiments was a modified Michelson interferometer, followed by a twophoton ionization chamber containing 100 Torr of NO gas (for further details see Ref. 25).

A series of autocorrelation curves was recorded with different values of $l$. Figures 3(b) and (c) show two traces obtained when $l$ was set to zero and $280 \mathrm{~cm}$, corresponding to no and optimum compensation, respectively. In agreement with our expectations the originally 430 -fs long pulse duration could be compressed to $90 \mathrm{fs}$, in a manner similar to its being compressed by the standard double prism pulse compressor.

\section{Limitations for GVD Compensated Arrangements}

It is seen from Eq. (7) that to get constant GVD across the beam at the position of the target, $l$ must be the same for different parts of the beam. Thus, the target to be pumped has to be parallel to the grating, otherwise spatially dependent broadening across the beam would occur. However, in this case the maximum tilt angle of the pulse front (or the maximum delay) is limited as shown in the following. If the length of the pumped volume of the target is $L$ (the same as that of the illuminated part of the grating), for exact synchronism between the pump pulse and the generated ASE in the pumped medium delay $D$ between the two marginal rays of the beam has to be

$$
D=\eta_{L} L,
$$

where $\eta_{L}$ is the group velocity index of the target material at the wavelength of the radiation to be generated. However, the delay created between these two rays is only

$$
D=L \sin \alpha \text { [see Fig. 2(b)], }
$$

where $\alpha$ is the angle of incidence of the beam at the grating. By comparing Eqs. (9) and (10),

$$
\sin \alpha=\eta_{L}
$$

is obtained. This equation cannot be fulfilled for values of $\eta_{L}>1$. Even for $\eta_{L}=1$ (e.g., $\mathrm{x}$-rays), $\alpha=90^{\circ}$ is obtained, where the efficiency of gratings is zero. The conclusion is that even though a single dispersive element is suitable to tilt the pulse front and to compress a positively chirped pulse at the same time, the pulse front tilt is not great enough for TWE, when a change of the pulse duration across the beamwidth is not allowed.

In earlier experiments, ${ }^{11-13,17,18}$ to fulfill the TWE condition, the target and the grating were not set par- allel but enclosed an angle, which necessarily led to spatially dependent GVD and, therefore, spatially dependent pulse broadening. Using the raytracing technique one can show that the spatially dependent GVD is proportional to target length $L$ and to the bandwidth of pump radiation $\Delta \lambda$. If the pump pulse is optimally compressed in the middle of the target and TWE is fulfilled for the center wavelength, the temporal broadening $\Delta t$ at the outer zones of the target due to the spatially dependent GVD can be expressed as

$$
\Delta t=\frac{\sin \varphi \tan ^{2} \gamma}{c \lambda \cos \beta} \frac{L}{2} \frac{\Delta \lambda}{2},
$$

where $\varphi$ is the angle between the grating and the target. Two numerical examples will show the importance of this fact. If the output pulses of the femtosecond $\mathrm{KrF}$ laser system ${ }^{25}$ are used for TWE of x-rays, the typical value of the group velocity index of the targets is $\eta=1$, if ASE in a dye cell has to be excited, $\eta=1.5$. The other parameters (grating constant $d$, angle between target and grating $\varphi$, incident angle of the beam at grating $\alpha$ ) were chosen to get optimum pulse compression in the middle of the target and perfect synchronism between the pump and the generated pulses (for their center wavelengths). For a 248-nm pump pulse, having a bandwidth of $1 \mathrm{~nm}$ and $500-\mathrm{fs}$ ( 100 -fs) uncompressed (compressed) pulse duration, and a $L=5-\mathrm{cm}$ long target, characterized by $\eta_{L}=1$, which encloses an angle $\varphi=47^{\circ}$ with the 1000 -lines $/ \mathrm{mm}$ grating (used at $\alpha=30^{\circ}$ ), the pulse broadening is $17 \mathrm{fs}$ at the outer zones, assuming perfect compensation and minimum $(100-\mathrm{fs})$ pulse duration in the middle of the target. However, for the same pump pulse and target length, but $\eta_{L}=1.5$ instead of 1 and $d=2 \times 10^{-4} \mathrm{~mm}, \alpha=80^{\circ}$ and $\varphi=30^{\circ}$ (the optimum parameters used for calculations), the pulse broadening is $\sim 150 \mathrm{fs}$, nearly an order of magnitude larger than in the first example. This is an indication that the error related to the nonparallel orientation of the grating and the target is increasing with increasing group velocity index of the target.

When using prisms ${ }^{14,16,19}$ to create a pulse front delay of this order of magnitude, the situation is more complicated as the result of the additional spatially dependent pulse broadening caused by GVD of the material of the prism(s) having spatially dependent thickness across the beam. ${ }^{26}$ For the above two numerical examples $(L=5$-cm long target with a group velocity index of $\eta=1$ and $\eta=1.5$ ) a pulse broadening of $160 \mathrm{fs}$ and $1.6 \mathrm{ps}$ is calculated, respectively.

It is seen from these examples that the error caused by the spatially dependent GVD is already comparable to the pulse duration when working with 100 -fs pulses and targets of several centimeters length with a group velocity index $\eta>1$.

\section{Possible Ways of Increasing the Tilt Angle of the Pulse Front at the Target}

\section{A. Using Immersion}

One way to increase the tilt angle of the pulse front is . to increase the absolute value of $D$. It is seen from Eq. 


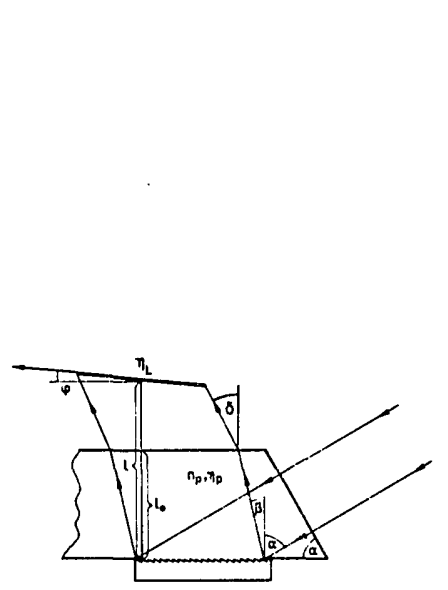

a)

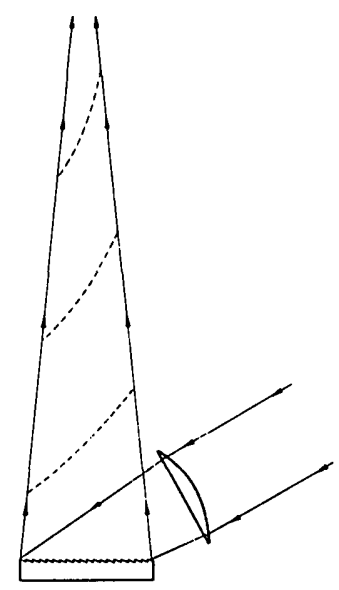

b)
Fig. 4. Possible ways to increase the pulse front tilt, using (a) immersion and (b) convergent beam (for details see text).

(10) that for parallel grating-target configuration and for a given target length $L$, the maximum delay of the pulse front between the two marginal rays is only determined by the incident angle $(D=L \sin \alpha)$ as shown in Fig. 2(b). However, if we use the experimental arrangement of Fig. 2 in immersion, and the incident and diffraction angles are left the same (by proper choice of the grating constant), the delay is multiplied by $\eta_{p}$ as

$$
D=\eta_{p} L \sin \alpha,
$$

where $\eta_{p}$ is the group velocity index of the immersion at the pump wavelength. Then Eq. (11) changes as

$$
\eta_{p} \sin \alpha=\eta_{L} \text {. }
$$

This equation can already be fulfilled for $\eta_{L}>1$ and $\alpha<90^{\circ}$ when $\eta_{p}>\eta_{L}$. With the use of immersion, one must be aware of the different value of the angular dispersion and the tilt angle of the pulse front, introduced by a grating. These quantities are given by the following equations:

$$
\begin{aligned}
& \frac{d \epsilon}{d \lambda}=\frac{\eta_{p}}{n_{p}^{2}} \frac{1}{d \cos \beta}, \\
& \tan \gamma=\frac{n_{p}}{\eta_{p}} \lambda \frac{d \epsilon}{d \lambda},
\end{aligned}
$$

where $n_{p}$ is the refractive index of the immersion at the pump wavelength. Figure 4(a) shows a possible experimental realization where the diffraction grating is surrounded by, e.g., a quartz block. For simplicity, the entrance window is chosen to be perpendicular to the incident pump beam, and the other surface of the quartz block (between the grating and the target) is parallel to the grating. In this case, the angular dispersion and the tilt angle of the pulse front after refraction on the boundary of the immersion changes to

$$
\frac{d \epsilon^{\prime}}{d \lambda}=\left(1-d \sin \alpha \frac{d n_{p}}{d \lambda}\right) \frac{1}{d \cos \delta},
$$

$$
\tan \gamma^{\prime}=\lambda \frac{d \epsilon^{\prime}}{d \lambda}
$$

where $\delta$ is the angle of the refracted beam [see Fig. 4(a)]. Since in this arrangement the path lengths in immersion are different for different parts of the beam, the spatially dependent GVD of the immersion must be considered. This can be compensated with an increased distance and with a properly chosen tilt angle $\varphi$ between the target and grating, resulting in a similar, spatially dependent GVD but of opposite sign. Using again the raytracing technique, one can show that $\sin \varphi$ has to be proportional to the GVD of the immersion at the pump wavelength:

$$
\sin \varphi=-\lambda \frac{d \eta_{p}}{d \lambda} \frac{\sin \alpha \cos (\delta+\varphi)}{\tan ^{2} \gamma^{\prime}},
$$

where $\gamma^{\prime}$ is the tilt angle of the pulse front at the position of the target. The importance of compensation is shown in a numerical example, where the arrangement in Fig. 4(a) is used for TWE of an $L=5-\mathrm{cm}$ long target characterized by $\eta_{L}=1.5$. The pump pulse is the same as used in the earlier numerical examples $\left(\lambda_{p}=248 \mathrm{~nm}, \Delta \lambda=1 \mathrm{~nm}, \tau_{\text {uncompressed }}=500 \mathrm{fs}\right.$, $\tau_{\text {compressed }}=100 \mathrm{fs}$ ). The 4000 lines $/ \mathrm{mm}$ density grating is in contact with a $l_{0}=20-\mathrm{mm}$ thick quartz block, characterized by a refractive index $n_{p}=1.5$, a group velocity index $\eta_{p}=1.6$, and a group velocity dispersion $d \eta_{p} / d \lambda=-2 \times 10^{-3}$ lines $/ \mathrm{nm}$ at the pump wavelength. For a parallel $(\varphi=0)$ grating-target configuration (when $\alpha=70^{\circ}, l=34 \mathrm{~mm}$ as optimum parameters used for calculations), the pulse broadening is $\sim 80 \mathrm{fs}$ at the outer zones of the target. However, if the target is tilted by $\varphi=16^{\circ}$ (when the optimum value of $\alpha=60^{\circ}$ and $l=45 \mathrm{~mm}$ ) the pulse broadening is only $0.5 \mathrm{fs}$.

This arrangement is well suited for pumping long targets due to the expansion of the beam by the grating. The main disadvantage of the arrangement is the spatially dependent GVD of the immersion, which has to be compensated for each configuration, and eventual nonlinear absorption ${ }^{32}$ when short wavelength lasers are used for pumping.

\section{B. Increase of the Pulse Front Tilt Through Demagnification}

The other way to increase the tilt angle $\gamma$ of the pulse front at the target is to leave the value of the delay $D$ between the marginal beams unchanged, but to decrease the beam size $L^{\prime}$ at the position of the target compared to that at grating $L$. Since

$$
\tan \gamma=\frac{D}{L^{\prime}}=\frac{L \sin \alpha}{L^{\prime}},
$$

a decrease in the beam size leads to an increase of $L / L^{\prime}$, resulting in an increase of the pulse front tilt. A simple realization is to use a convergent beam between the grating and the target. This can be achieved by a focusing element positioned either before or after the grating. If focusing is done before the grating, the angular dispersion of the grating is not influenced, but the convergent angle of the diffracted beam $\left(\Psi^{\prime}\right)$ changes, as can be calculated from the equation 


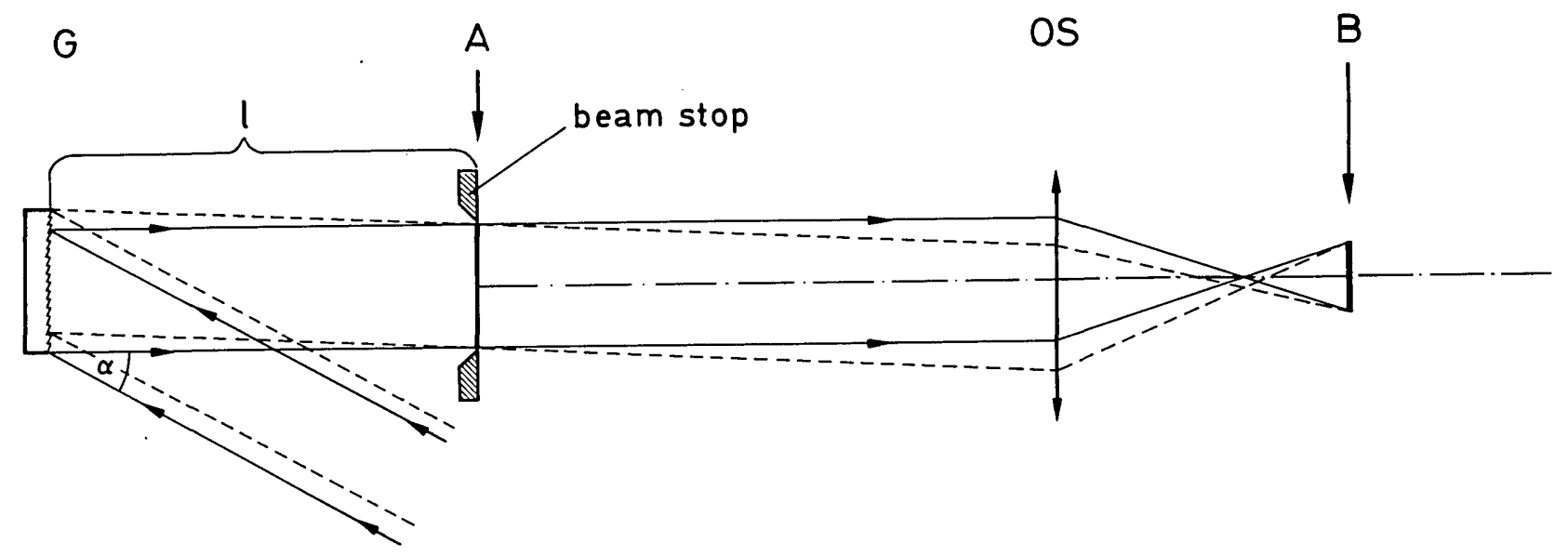

Fig. 5. Optimized compressor-TWE arrangement using optical imaging.

$$
\Psi^{\prime}=\left(\frac{\cos \alpha}{\cos \beta}\right) \Psi,
$$

where $\Psi$ is the convergent angle of the input beam.

If a lens with focal length $f$ is positioned after the grating,the angular dispersion changes to

$$
\frac{d \epsilon^{\prime}}{d \lambda}=-\left(\frac{a}{f}-1\right) \frac{d \epsilon}{d \lambda},
$$

where $a$ is the distance between the lens and the grating. Note that both the magnitude and the sign of the angular dispersion can be influenced by proper choice of $a$ (see also Ref. 33).

Figure 4(b) shows that variant where the focusing element, a cylindrical lens, is used before the grating. The increasing tilt angle of the pulse front (shown by dashed lines in the figure) is also indicated, as the beam diameter decreases. This arrangement is simple and well suited for pumping short targets.

The main drawback of this approach is related to the curved phase and pulse fronts at the target due to the convergent beam used here. This can be avoided, and perfect compensation is expected to be achieved when the beam size is decreased by the use of optical imaging (Fig. 5).

It has been shown above that there is a plane parallel to the grating, where exact pulse compression can be obtained, accompanied with a tilt of the pulse front, which unfortunately is not enough for direct TWE. Let us denote this plane by $A$ (Fig. 5). If a demagnified picture of plane $A$ is made at plane $B$ by an ideal optical system $O S$, the tilt angle of the pulse front in plane $B$ can be arbitrarily increased. Since optical imaging leaves the absolute value of the delay between the two marginal ways unchanged, the increase of the tangent of the pulse front tilt can be calculated from demagnification $M$ as

$$
\tan \gamma^{\prime}=M \tan \gamma,
$$

where $\gamma^{\prime}$ and $\gamma$ are the tilt angles of the pulse fronts in planes $B$ and $A$, respectively. In this arrangement the direction of propagation of the beam can be arbitrarily chosen with respect to planes $A$ and $B$. For simplicity let us assume the beam to be perpendicular to planes $A$ and $B$, therefore, because of the necessary parallelism of these planes to the grating, the diffracted beam is also perpendicular to the surface of the grating $(\beta=0)$.

In Fig. 5 at the position of plane $A$, a beam stop is used to select the middle part of the beam, containing all spectral components as discussed earlier. For two wavelength components (the red component is shown by a dashed line, the blue one by a solid line), those incident rays are indicated which became marginal rays after passage through the beam stop. Then, assuming a target in plane $B$, having a group velocity index of $\eta_{L}$ at the wavelength of radiation to be generated, a pulse front tilt of $\gamma^{\prime}$ needed for $\mathrm{TWE}^{11}$ is given by

$$
\tan \gamma^{\prime}=\eta_{L}
$$

as can be derived from Eq. (9). From Eqs. (23) and (24), one gets

$$
\tan \gamma=\frac{\eta_{L}}{M}
$$

for plane $A$. From Eqs. (4) and (2), substituting $\beta=0$, one can calculate the pulse front tilt introduced by the grating as

$$
\tan \gamma=\frac{\lambda}{d} .
$$

Comparing Eqs. (25) and (26),

$$
\frac{M}{d}=\frac{\eta_{L}}{\lambda}
$$

is obtained, but at the same time the grating equation

$$
d \sin \alpha=\lambda
$$

must also be fulfilled. The necessary value of $l$ can be calculated from Eq. (7) using the angular dispersion of the grating, with the $\beta=0$ condition, as

$$
l=\frac{c d^{2}}{\lambda} \frac{d T}{d \lambda} \text {. }
$$


From Eqs. (27)-(29) all the parameters of the pump arrangement can easily be determined: using Eqs. (27) and (28) for a given pump wavelength and for a target (characterized by $\eta_{L}$ ), a practical value for grating constant $d$ can be chosen. Even for different values of $d$, Eqs. (27) and (28) can be fulfilled by proper choice of the demagnification $M$ and the angle of incidence $\alpha$. The value of $l$ corresponding to optimum pulse compression can be calculated from Eq. (29) for an incoming chirped pulse, whose chirp is characterized by $d T / d \lambda$.

For example, using the same pump pulses as in the earlier examples, a 2442 -lines $/ \mathrm{mm}$ grating (used at $\alpha=$ $37.3^{\circ}$ and $\beta=0^{\circ}$ ) gives perfect pulse compression in plane $A$ which must be $10.1 \mathrm{~cm}$ away from the grating. Then, using different demagnifications, exact synchronism can be achieved for different targets $(M=$ 1.65 and 2.48 for $\eta_{L}=1$ and 1.5, respectively). In this way, for any pump wavelength and any target group velocity index, the experimental parameters can be chosen in a simple manner and exact TWE with optimum pulse compression on the target is expected.

In the position of $O S$ an optical image system is required to be used, which is highly corrected against spherical and chromatic aberrations (and therefore against pulse front distortion). ${ }^{29,30}$ Either an achromat or-especially in the UV-reflective optics are recommended. ${ }^{29}$ The additional advantage of the demagnification applied in this method is that the size of the pumped volume at plane $B$ can be better controlled, and a diffraction-limited picture can easily be created.

The efficiency of the arrangement is mainly characterized by the efficiency of the single grating used there. Considering that the transmission of the image system can be very high, and coupling of the pump energy into the target, resulting from the free choice of the incident angle, is optimal, ${ }^{34}$ a very high overall efficiency is expected. This picture is especially attractive when considering that in the earlier cases separate arrangements had to be used as a compressor and as a TWE arrangement consisting of more components.

An interesting feature of this combined arrangement is that the compressed pulse is only present at planes $A$ and $B$; at other points of the system compression is not complete [as also seen from Fig. 2(b), by substituting the target by plane $A]$. Between the grating and $O S$ the temporal dispersion constant can be described by Eq. (7). After OS, both the angular dispersion and the pulse front tilt become spatially dependent. The spatial dependence of the temporal dispersion constant can be described by

$$
\frac{d T}{d \lambda}=\left(\frac{d T}{d \lambda}\right)_{0}-\left(\frac{1}{f}-\frac{1}{x}\right)^{-1} \frac{\lambda}{c}\left(\frac{d \epsilon}{d \lambda}\right)^{2},
$$

where $x$ is the distance from $O S$ and $f$ is the focal length of OS. The temporal dispersion constant of the position of $O S$ is described by $(d T / d \lambda)_{0}$. This equation was deduced neglecting the diffraction effects on finite apertures, therefore it is only valid for points away from the focus.
Generally, the final amplifier and the target should not be separated by other low efficiency components (pulse compressor, TWE schemes) to optimize the energy incident on the target. On the other hand, amplification is not recommended to be done after pulse compression, since in most cases it leads to selective amplification of the leading temporal wings of the compressed pulse, resulting in significant temporal broadening. ${ }^{26}$ In the present system, both requirements (optimization for the highest efficiency, avoidance of temporal broadening)seem to be fulfilled if the amplifier is positioned between $A$ and $O S$, where the pulse is not fully compressed and the amplifier is only separated from the target by a high efficiency lens (or mirror) $O S$. This can result in optimum pulse compression with very high efficiency and makes it possible to simulate the conventional chirped pulse amplification (CPA) technique ${ }^{35}$ in a simple manner. An experimental study of this method will be presented elsewhere. ${ }^{36}$

For the above reasons this arrangement could meet wide applications in the field of short pulse generation and in target experiments. Experimental studies and future aspects of this experimental realization of combined pulse compression and TWE for target experiments will be presented in a forthcoming paper.

\section{Conclusion}

We have shown that a single dispersive element can be used both for pulse front tilt and for pulse compression and is, therefore, ideally suited for TWE of targets with positively chirped pulses. We derived a formula for the negative GVD of such a device, which is found to be exactly the same as that of the double grating or double prism compressor. In this sense, the second dispersive element of the generally used compressors is found necessary only to make the pulse front again parallel to the phase front and to preserve the negative GVD. It is shown that in many applications the use of this second element is not needed, resulting in an improved efficiency. These considerations were checked by an experiment, where pulse duration measurements, using the general autocorrelation technique, were performed after a single dispersive element. The same results are obtained as with conventional pulse compressors.

If spatially dependent GVD must be avoided across the beam, the target must be parallel to the grating used as a dispersive element. It is shown that in this case no direct TWE of any target is possible because of the limited tilt angle of the pulse front. Three possibilities are shown to overcome this problem: using immersion, a convergent beam, and optical imaging. The advantages, the special consequences and also the limitations of each method are discussed. It is shown that with the use of the last approach, one can have an exact, generally applicable and efficient method for TWE of any target, accompanied by perfect temporal compression of the pulse just at the position of the target. 
The authors wish to thank F. P. Schafer for his useful comments and $\mathrm{H}$. Gerhardt for critical reading of the manuscript. This work was supported by the Bundesministerium für Forschung und Technologie and the Deutsche Forschungsgemeinschaft by the Gottfried-Wilhelm-Leibniz-Program and by the OTKA Foundation of the Hungarian Academy of Sciences.

Sandor Szatmari and Peter Simon are both on leave from JATE University, Hungary.

\section{References}

1. M. E. Mack, "Superradiant Traveling-Wave Dye Laser," Appl. Phys. Lett. 15, 166-168 (1969).

2. M. M. Malley and P. M. Rentzepis, "Picosecond Time-Resolved Stimulation Light Emission," Chem. Phys. Lett. 7, 57-60 (1970).

3. M. R. Topp, P. M. Rentzepis, and R. P. Jones, "Time-Resolved Picosecond Emission Spectroscopy of Organic Dye Lasers," Chem. Phys. Lett. 9, 1-5 (1971).

4. C. Lin, T. K. Gustafson, and A. Dienes, "Superradiant Picosecond Laser Emission from Transversely Pumped Dye Solution," Opt. Commun. 8, 210-215 (1973).

5. A. N. Rubinov, M. C. Richardson, K. Sala, and A. J. Alcock, "Generation of Single-Picosecond Dye Laser Pulses Using Oneand Two-Photon Traveling-Wave Excitation," Appl. Phys. Lett. 27, 358-360 (1975).

6. G. R. Fleming, A. E. W. Knight, J. M. Morris, R. J. Robbins, and G. W. Robinson, "Picosecond Spectroscopic Studies of Spontaneous and Stimulated Emission in Organic Dye Molecules," Chem. Phys. 23, 61-70 (1977).

7. W. Falkenstein, A. Penzkofer, and W. Kaiser, "Amplified Spontaneous Emission in Rhodamine Dyes: Generation of Picosecond Light Pulses and Determination of Excited State Absorption and Relaxation," Opt. Commun. 27, 151-156 (1978).

8. A. Penzkofer and W. Falkenstein, "Theoretical Investigation of Amplified Spontaneous Emission with Picosecond Light Pulses in Dye Solution," Opt. Quantum Electron. 10, 399-423 (1978).

9. A. Penzkofer and J. Wiedmann, "Orientation of Transition Dipole Moments of Rhodamine 6G Determined by Excited State Absorption," Opt. Commun. 35, 81-86 (1980).

10. A. N. Rubinov, B. A. Bushuk, A. A. Murav'ov, and A. P. Stupak, "Picosecond Spectroscopy of Intermolecular Interactions in Dye Solutions," Appl. Phys. B 30, 99-104 (1983).

11. Zs. Bor, S. Szatmari, and A. Muller, "Picosecond Pulse Shortening by Traveling Wave Amplified Spontaneous Emission," Appl. Phys. B 32, 101-104 (1983).

12. H. J. Polland, T. Elsaesser, A. Seilmeier, and W. Kaiser, "Picosecond Dye Laser Emission in the Infrared Between 1.4 and 1.8 $\mu \mathrm{m}$," Appl. Phys. B 32, 53-57 (1983).

13. S. Szatmari and F. P. Schafer, "A Tunable, Highly Monochromatic Picosecond Light Source," Opt. Commun. 49, 281-284 (1984).

14. Zs. Bor and B. Racz, "Group Velocity Dispersion in Prism and Its Application to Pulse Compression and Traveling-Wave Excitation," Opt. Commun. 54, 165-170 (1985).

15. J. Klebniczki, Zs. Bor, and G. Szabo, "Theory of Traveling-Wave Excited Amplified Spontaneous Emission,” Appl. Phys. B 46, 151-155 (1987).
16. J. Hebling, J. Klebniczki, P. Heszler, Zs. Bor, and B. Racz, "Traveling-Wave Amplified Spontaneous Emission Excited in a Prismatic Geometry," Appl. Phys. B 48, 401-403 (1989).

17. T. Elsaesser, H. J. Polland, A. Seilmeier, and W. Kaiser. "Narrow-Band Infrared Picosecond Pulses Tunable Between 1.2 and $1.4 \mu \mathrm{m}$ Generated by a Traveling-Wave Dye Laser," IEEE J. Quantum Electron. QE-20, 191-194 (1984).

18. H. Lobentanzer and T. Elsaesser, "Theoretical and Experimental Analysis of Infrared Dye-Laser Action in a Traveling-Wave Pumping Geometry," Appl. Phys. B 41, 139-145 (1986).

19. J. Hebling and J. Kuhl, "Generation of Femtosecond Pulses by Traveling-Wave Amplified Spontaneous Emission," Opt. Lett. 14, 278-280 (1989).

20. F. P. Schafer, "On Some Properties of Axicons," Appl. Phys. B 39, 1-8 (1986).

21. F. P. Schafer, "Die Erzeugung ultrakurzer Laserimpulse im Ultraviolett und Röntgenbereich," Phys. Bl. 42, 283-288 (1986).

22. C. P. J. Barty, D. A. King, G. Y. Yin, K. H. Hahn, J. E. Field, J. F. Young, and S. E. Harris, "12.8-eV Laser in Neutral Cesium," Phys. Rev. Lett. 61, 2201-2204 (1988).

23. W. T. Silfvast and O. R. Wood II, "Simple Efficient TravelingWave Excitation of Short-Wavelength Lasers Using a Conical Pumping Geometry," Opt. Lett. 14, 18-20 (1989).

24. S. Szatmari, F. P. Schafer, E. Muller-Horsche, and W. Muckenheim, "Hybrid Dye-Excimer Laser System for the Generation of $80 \mathrm{fs}, 900 \mathrm{GW}$ Pulses at $248 \mathrm{~nm}$," Opt. Commun. 63, 305-309 (1987).

25. S. Szatmari and F. P. Schafer, "Simplified Laser System for the Generation of $60 \mathrm{fs}$ Pulses at $248 \mathrm{~nm}$," Opt. Commun. 68, 196202 (1988).

26. S. Szatmari, G. Kuhnle, J. Jasny, and F. P. Schafer, "KrF Laser System with Corrected Pulse Front and Compressed Pulse Duration," Appl. Phys. B 49, 239-244 (1989).

27. O. E. Martinez, J. P. Gordon, and R. L. Fork, "Negative GroupVelocity Dispersion Using Refraction," J. Opt. Soc. Am. A 1, 1003-1006 (1984).

28. E. B. Treacy, "Optical Pulse Compression with Diffraction Gratings," IEEE J. Quantum Electron. QE-5, 454-458 (1969).

29. S. Szatmari and G. Kuhnle, "Pulse Front and Pulse Duration Distortion in Refractive Optics, and Its Compensation," Opt. Commun. 69, 60-65 (1988).

30. Zs. Bor, "Distortion of Femtosecond Laser Pulses in Lenses," Opt. Lett. 14, 119-121 (1989); Zs. Bor, "Distortion of Femtosecond Laser Pulses in Lenses and Lens Systems," J. Mod. Opt. 35, 1907-1918 (1988).

31. P. Simon, H. Gerhardt, and S. Szatmari, "Simple Method for Temporal Study of Subpicosecond Distributed Feedback Dye Lasers," Opt. Commun. 71, 305-310 (1989).

32. A. J. Taylor, R. B. Gibson, and J. P. Roberts, "Two-Photon Absorption at $248 \mathrm{~nm}$ in Ultraviolet Window Materials," Opt. Lett. 13, 814-916 (1988).

33. J. P. Gordon and R. L. Fork, "Optical Resonator with Negative Dispersion," Opt. Lett. 9, 153-155 (1984).

34. R. Fedosejevs, R. Ottmann, R. Sigel, G. Kuhnle, S. Szatmari, and F. P. Schafer, "Absorption of Subpicosecond Ultraviolet Laser Pulses in High-Density Plasma," Appl. Phys. B 50, 79-99 (1990).

35. P. Maine, D. Strickland, P. Bado, M. Pesssot, and G. Mourou, "Generation of Ultrahigh Peak Power Pulses by Chirped Pulse Amplification," IEEE J. Quantum Electron. QE-24, 398-403 (1988).

36. S. Szatmari, P. Simon, and H. Gerhardt, "Generation of $135 \mathrm{fs}$ Pulses of Variable Pulse Front Tilt by Spatially-Evolving Chirped-Pulse Amplification at $248 \mathrm{~nm}$," Opt. Commun., in press. 